\title{
Hafnia alvei bacteremia following bronchopneumonia in an eleven month old child: a case report from a tertiary care hospital in Nepal
}

\author{
Nisha Baral, Niranjan Nayak*, Rajani Shrestha, Ranjana Parajuli, Deependra Hamal, Dhrama \\ Raj Bhatta, Supram Gowda, Shishir Gokhale
}

Department of Microbiology

Manipal College of Medical Sciences, Pokhara, Nepal

Correspondence E-mail: *niruni2000@yahoo.com

\begin{abstract}
Hafnia alvei one of the members of family Enterobacteriaceae are related to infections that are uncommon. Believed to be an opportunistic pathogen, it is responsible for causing invasive infections in debilitated elderly people with underlying illnesses. Hafnia alvei infections, however, are extremely rare among immunocompetent neonates. We hereby, report a case of Hafnia alvei bacteremia following bronchopneumonia in an eleven month old male child who was apparently healthy and immunocompetent. To the best of our knowledge, this case report is the first of its kind from Nepal.
\end{abstract}

Keywords: Hafnia alvei, Bronchopneumonia, Bacteremia

\section{Introduction}

Hafnia alvei formerly designated as Enterobacter hafniae is currently the sole species in the genus Hafnia (Donnenberg, 2010). It is one of the members in the family Enterobacteriaceae, being a gram negative motile non-lactose fermenting facultative anaerobic bacillus. It has both saprophytic as well as parasitic existence in mammals, birds, reptiles, fish, soil, water, sewage, and food (Gunthard et al.,1996; Farmer, 2003; Harmer et al.,1985). H. alvei is often regarded as a commensal in the human gastrointestinal tract (Gunthard et al., 1996). However, occasional infections due to this organism were reported in patients with severe underlying conditions like malignancies, trauma or following surgery (Tille., 2014).

$H$. alvei, though, an uncommon human pathogen, has received much attention over the past decade due to its possible association with gastroenteritis and rare isolation from clinical specimens like urine, body fluid, tissues and catheters (Gunthard et al.,1996; Washington et al., 1971). However, there have been relatively few cases of Hafnia bacteremia reported in the literature.

It is believed that Hafnia alvei is an opportunistic pathogen responsible for causing invasive disease only amongst debilitated patients (Casanova-Roman et al., 2004; Ginsberg et al., 1988). Isolation of this organism is an unusual finding from otherwise healthy immunocompetent individual. In view of the above, we report a case of bacteremia following bronchopneumonia in an eleven month old child without any immunosuppressive predisposition.

\section{Case Report}

A 11-month-old infant presented to the emergency department at Manipal Teaching Hospital, Pokhara with cough of approximately 
48 hours and noisy breathing of 24 hours' duration. Upon enquiry from the mother, it was noted that cough was acute in onset and nonproductive in nature. Gradually, the infant developed noisy fast breathing. The mother revealed that there was no history of fever or vomiting. The infant was passing urine and stool normally as per daily basis with good feeding response. Shortly before, the patient was treated at other medical facility with stat dose of injection dexamethasone and injection gentamicin. In spite of this, his condition worsened and he was referred to Manipal Teaching Hospital (MTH), a tertiary care teaching hospital in western Nepal.

The infant's initial physical examination was notable for a body temperature of $98.6^{\circ} \mathrm{F}$, respiratory rate 68 breaths $/ \mathrm{min}$, heart rate 128 beats/min. The infant appeared acutely ill and his respiratory examination displayed presence of bilateral diffuse wheeze with intercostal recession. His past history revealed that he was delivered by an uncomplicated vaginal delivery following a gestation period of 37 weeks, progressed with normal developmental milestones and was vaccinated as per EPI schedule of Nepal.
On arrival at emergency department with the above findings, the patient was shifted to paediatric ICU. Subsequent to all routine investigations, he was empirically treated with injection cefotaxime $450 \mathrm{mg}$ IV tds along with IV infusion of Isolyte two pints over 24 hours, oxygen inhalation and nebulisation. Tracing the reports, the complete blood cell count revealed a WBC count of $19 \times 10^{3} / \mu \mathrm{L}$, featuring $\quad 81.0 \% \quad$ neutrophils, $19.0 \%$ lymphocytes. The CRP level was $96 \mathrm{mg} / \mathrm{dl}$ with normal electrolytes and renal profile. X-ray chest showed the features of pneumonia and the initial diagnosis of the case was bronchopneumonia. The blood sent for culture and sensitivity in $\mathrm{BHI}$ (brain hear infusion) broth revealed turbidity after 96 hours of incubation at $37^{\circ} \mathrm{C}$.

For complete identification, subculture from $\mathrm{BHI}$ broth was done on sheep Blood Agar (BA) and MacConkey Agar (MAC) and Nutrient Agar (NA). After 24 hours of incubation, the BA plate showed growth of small, round, smooth, opaque, non-haemolytic colonies (Figure 1A).

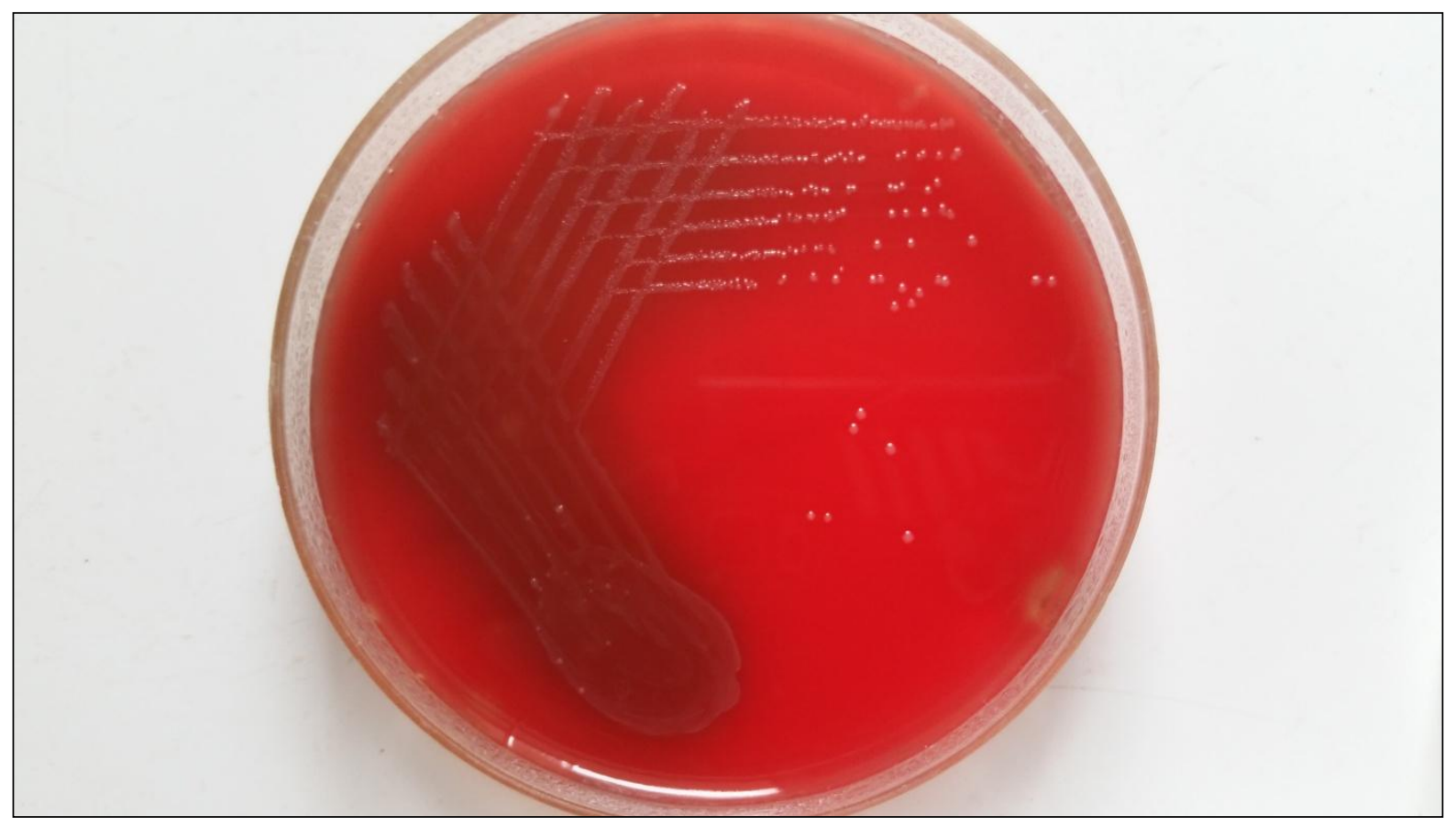

Figure 1 A: Non-hemolytic colonies of $H$. alvei on blood agar plate 
MAC plate showed growth of small, round, smooth, non-lactose fermenting bacteria (Figure 1 B). Nutrient agar plate exhibited production of greyish convex colonies, 1-2 mm in diameter (Figure $1 \mathrm{C}$ ). Gram staining from colonies from all three i.e. BA, MAC and NA plates revealed same microscopic morphology showing gram negative, short, nonsporing bacilli (Figure 2).

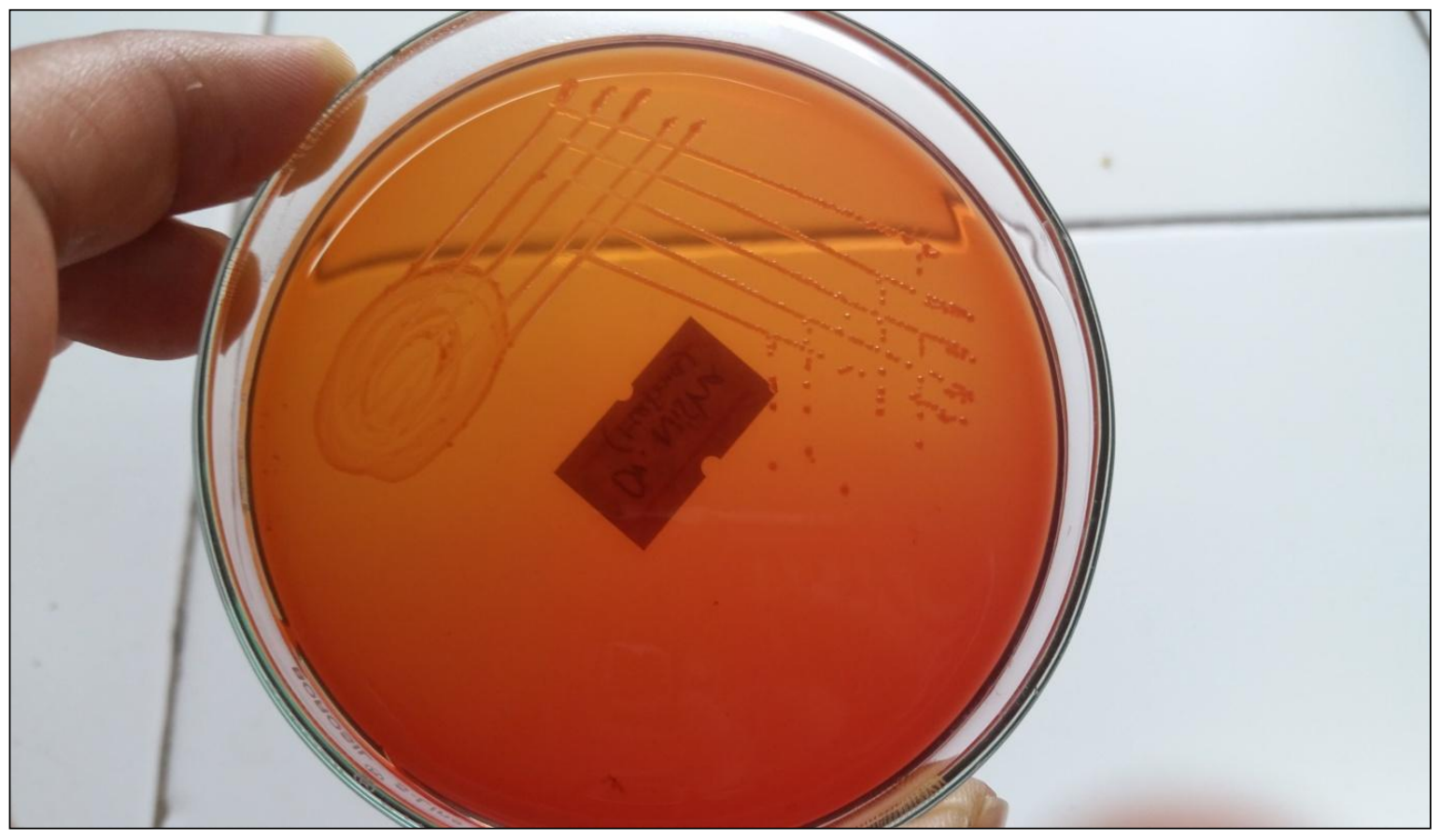

Figure $1 \mathrm{~B}$ : MacConkey Agar plate showing non-lactose fermenting colonies

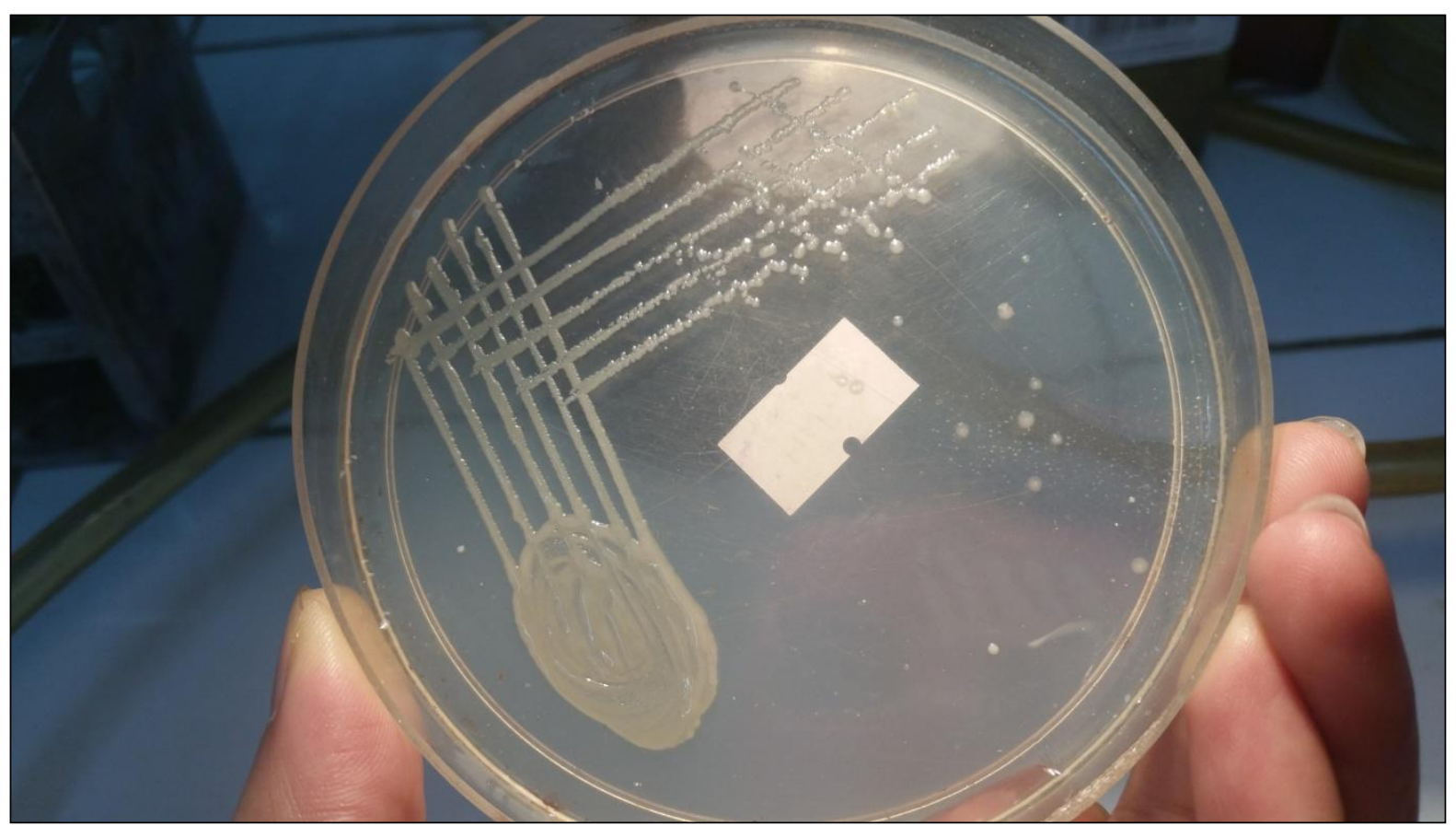

Figure 1 C: Greyish convex colonies of $H$. alvei on NA 


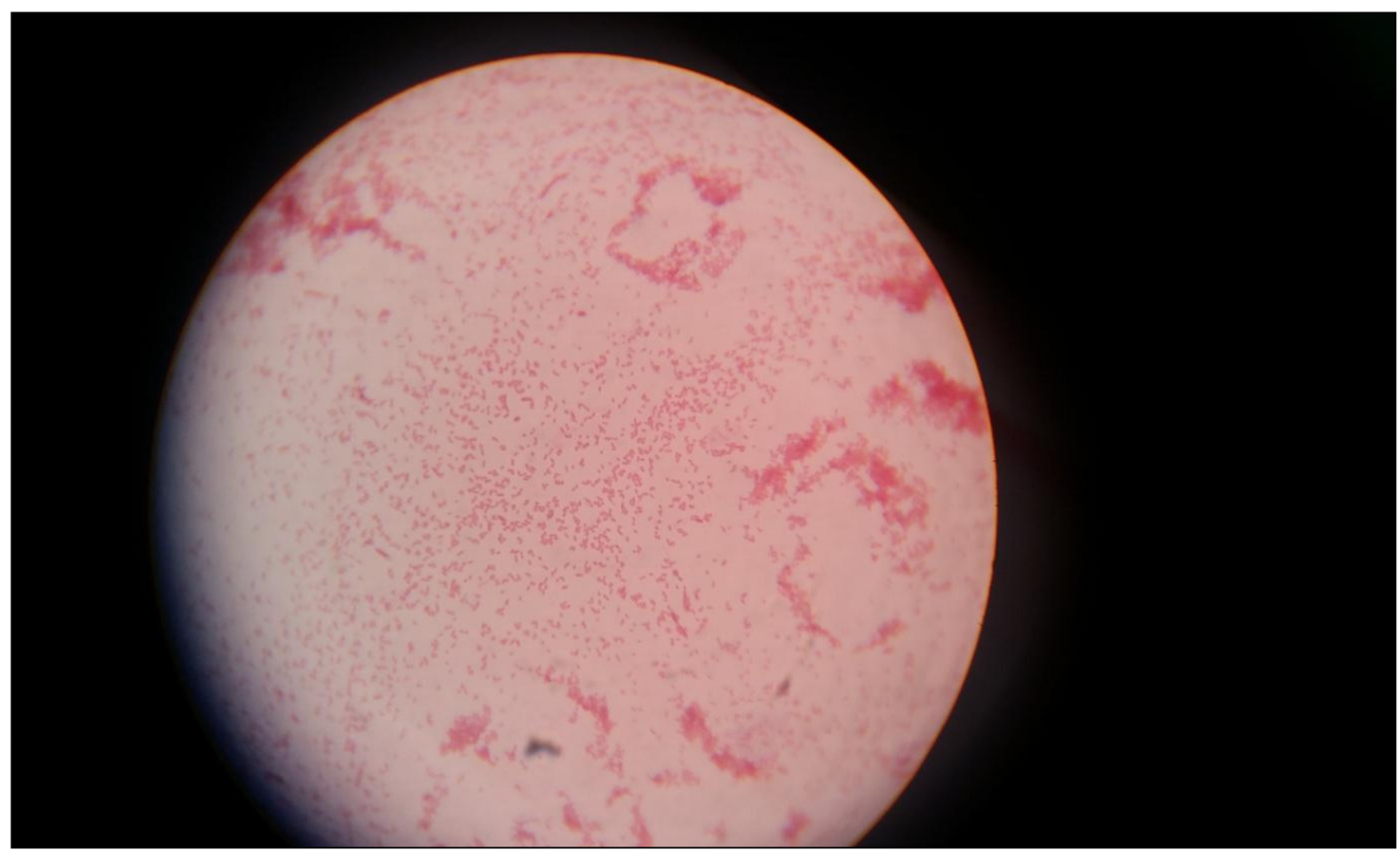

Figure 2: Gram stain showing short gram negative bacilli

The organism fermented glucose, and mannitol but not lactose with production of acid and gas. It was non-motile at $37^{\circ} \mathrm{C}$ but showed motility at room temperature at $30^{\circ} \mathrm{C}$ as observed in SIM (sulphur-indole motility) test. It was indole negative, did not produce hydrogen sulphide $(\mathrm{H} 2 \mathrm{~S})$ gas and was oxidase negative. It was catalase positive and reduced nitrate to nitrite, did not utilize citrate at $37^{\circ} \mathrm{C}$ but grew on Simmon's citrate medium at $22^{\circ} \mathrm{C}$ and did not hydrolyse urea (Figure 3). Further, the isolate was VP negative, MR positive, decarboxylated ornithine and lysine. It was, thus, identified as Hafnia alvei based on the above mentioned characteristics (Tille., 2014; Holmes et al.,1990). The organism was sensitive to cefepime, ceftriaxone, cefotaxime, amikacin, gentamicin, imipenem, ciprofloxacin, cefoperazone-sulbactum and polymixin B but resistant to ampicillin and amoxicillin/clavulanic acid.

With prompt management that included oxygen inhalation, nebulisation, infusion with normal saline and antibiotic cefotaxime patient showed marked clinical improvement. After one week of therapy, patient improved markedly, was asymptomatic and was discharged from the hospital.

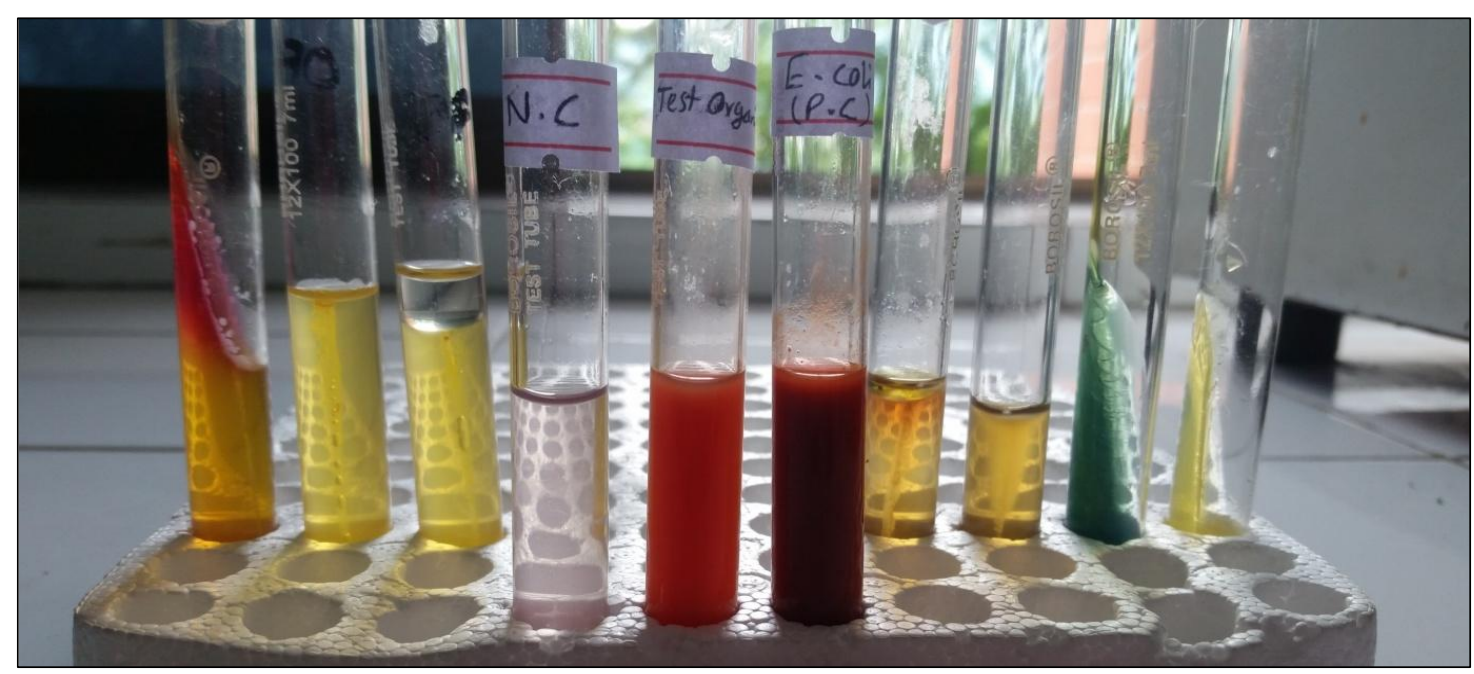

Fiqure 3: Biochemical reactions of Hafnia alvei 


\section{Discussion}

To the best of our knowledge, this is the first case of invasive $H$. alvei infection reported from Nepal. H. alvei, being a normal resident flora of human gut, is often isolated from the faeces of healthy individuals. However, reports regarding $H$ alvei causing invasive infections are scanty. Jennis and McCarthy. (19670 first discovered the link of $H$. alvei with human illness. Janda and Abbot. (2006) also provided strong evidence that $H$. alvei could cause extra-intestinal infections.

To date, there were few reports regarding Hafnia bacteremia in the paediatric age group with underlying immunocompromised states but there were hardly any documentation on Hafnia bacteremia in immunocompetent neonates (Washington et al., 1971; Janda and Abbott., 2006).

The present case was a 11 month old male child who was apparently healthy and immunocompetent. Since the child was febrile, his blood sample was collected and subjected to culture and sensitivity, which grew $H$ alvei. No other clinical material was, however, processed for any microbiological investigation.

The pathogenesis of invasive $H$ alvei infection is obscure. However, gastrointestinal tract has frequently been implicated as the source (Lee et al., 2007). In the event of any breach in the continuity of gastrointestinal mucosa, such as abdominal wound or abdominal surgery, it would be reasonable to speculate gastrointestinal tract as the main source of blood stream invasion (Jennis \& Mccarthy., 1967). Mojtabaee et al. (1978) in the past reported that the main portal of entry for $H$. alvei bacteraemia could be the gastrointestinal tract and meningitis was a subsequent event following the bacteraemic episodes in their patients. Others (Janda and Abbott., 2006) reported a series of cases of Hafnia bacteaemia about half of which had antecedent immunocompromised conditions including malignancies, HIV infection and liver transplant, and postulated that the presumed origin of infection in their series was either gastrointestinal tract or the respiratory tract.
In the present case, the child had bronchopneumonia which could be the primary focus leading secondarily to bacteraemia. Since, the organism was isolated from blood as a pure growth, it was considered to be the sole pathogen causing bacteremia in the child presumably following bronchopneumonia (Janda and Abbott. 2006). In a similar case study, Gunthard and Pennakamp. (1996) concluded that the only identified pathogen in their cases of septicaemia and peritonitis happened to be $H$ alvei, and in case of peritonitis $H$ alvei was isolated as pure growth both from peritoneal fluid and blood. Earlier workers (Sakazaki et al., 1986) too advocated that the likely portal of entry of $H$ alvei into the blood stream was the gastrointestinal tract, where it was considered to be a commensal.

On conventional routine culture, $H$. alvei often can be mistaken for H2S-negative Salmonella or could be confused with/misidentified as either Enterobacter or Serratia (Janda and Abbott., 2006) . Thus, one should have high index of suspicion and correct approach should be initiated for its accurate identification.

Recommended treatment of $H$. alvei infections include third generation cephalosporins, in combination with aminoglycosides, as this organism is invariably resistant to ampicillin, first generation cephalosporins and amoxycillin/clavulanic acid (Gunthard and Pennekamp., 1996; Lee CH., 2007), due to its inducible and constitutive beta-lactamase production.In present case, the organism was sensitive to third generation cephalosporins, and the child responded fairly well to cefotaxime regime with other supportive therapy and was discharged after getting fully recovered.

\section{Conclusion}

Hafnia alvei is among the rare pathogens causing bacteraemia, that was encountered in a tertiary care hospital like ours. This case emphasises the possibility of an extra intestinal invasive condition such as bacteraemia or bronchopneumonia. Thus, isolation of such unusual pathogens from the blood stream is often challenging, and one 
should have high index of suspicion for $\mathrm{H}$ alvei extra intestinal infection in young infants who feature no apparent underlying disease.

\section{References}

Casanova-Roman, M., Sanchez-Porto, A. \& Casanova-Bellido, M.(2004). Late-onset neonatal sepsis due to Hafnia alvei. Scandinavian Journal of Infectious Diseases. 3691, 70-2.

Donnenberg, M.S (2010). Enterobacteriaceae. In: GL.Mandell. Bennett. J.E. Dolin. R, ed. Mandell, Douglas, and Bennett's Principles and Practice of Infectious Diseases. $7^{\text {th }}$ edition, Philadelphia: Churchill-Livingstone. 2815-33.

Farmer, J. J III., Davis, B. R., HickmanBrenner, F. W., McWhorter, A., Huntley-Carter, G.P., Asbury, M. A. et al. (1985). Biochemical identification of new species and biogroups of Enterobacteriaceae isolated from clinical specimens. Journal of Clinical Microbiology. 21(1), 46-76.

Farmer, J.J. III (2003). Enterobacteriaceae: introduction and identification. In: PR. Murray, Baron,E. J., Jorgensen,J. H., Pfaller, M. A.,Tolken, R. H, ed. Manual of clinical microbiology $8^{\text {th }}$ edition. ASM, Washington D.C., 636-53.

Ginsberg, H.G., Goldsmith, J.P. (1988). Hafnia alvei septicemia in an infant with necrotizing enterocolitis. Journal of Perinatology.8(2). pp122-3.

Gunthard, H. \& Pennekamp, A. (1996). Clinical significance of extraintestinal Hafnia alvei isolates from 61 patients and review of the literature. Clinical Infectious Disease. 22(6), 1040-5.

Holmes, B., Gross, R.J. (1990). Coliform bacteria; various other members of the
Effective treatment should be carefully be guided based on the preliminary antimicrobial susceptibility testing.

Enterobacteriaceae: In: Parker MT. Duerden B.I, ed. Topley and Wilson's Principles of bacteriology, virology and immunity vol III $8^{\text {th }}$ edition. London: Edward Arnold., 415-41.

Janda, J.M. \& Abbott, S.L.(2006). The genus Hafnia: from soup to nuts. Journal of Clinical Microbiology. 19(1), 12-8.

Jennis, F. \& Mccarthy, S.W. (1967). Hafnia: an unusual cause of postoperative gram-negative bacteraemia. Medical Journal of Australia. 1(6), 286-7

Liu, C.H., Lin,W.J., Wang, C.C., Lee, K-long., Tsai M.C.(2007).Young infant sepsis combined with urinary tract infection due to Hafnia alvei. Journal of Formos Medical Association. 106(suppl 3), S 39-43.

Mojtabaee,A.\& Siadati, A.(1978). Enterobacter hafnia meningitis. Journal of Paediatrics. 939(6), 1062-3.

Sakazaki, R. \& Genus, I.X.(1986). Hafnia: In: Holt, J.G, ed. Bergey's manual of systematic bacteriology 7th edition. Baltimore Williams and Wilkins., 484-6.

Tille, P.M.(2014). Enterobacteriaceae. In: Bailey and Scott's Diagnostic microbiology. $13^{\text {th }}$ edition, South Dakota, Elsevier, 307-28.

Washington, J.A II., Birk, R.J., Ritts, R.E Jr.(1971). Bacteriologic and epidemiologic characteristics of Enterobacter hafniae and Enterobacter liquefaciens. Journal of Infectious Diseases.124 (4), 379-86. 